\title{
Posicionamento em Ambientes Internos nas Redes 5G: um Auxílio para Deficientes Visuais
}

\author{
Paulo Francisco da Conceição e Flávio Geraldo Coelho Rocha
}

\begin{abstract}
Resumo-Localizar o dispositivo móvel em uma rede celular pode ter diversas aplicações, como auxiliar na locomoção dos deficientes visuais, proporcionando maior qualidade de vida. Embora o GPS (Global Positioning System) proporcione uma boa precisão para localização, em ambientes internos ele perde a linha de visada direta com os satélites, degradando a precisão. As redes móveis de quinta geração apresentam características que podem melhorar a precisão de localização do dispositivo. Este trabalho apresenta uma abordagem para o pré-processamento da localização em ambientes internos usando parâmetros determinados a partir de uma transmissão em rede $5 \mathrm{G}$ e formulações geométricas de uma propagação em ambiente NLoS (Non-Line-of-Sight).
\end{abstract}

Palavras-Chave-Deficientes Visuais, Localização, 5G.

Abstract-The localization of the mobile device on a cellular network can have several applications, such as assisting the locomotion of visually impaired persons, providing greater quality of life. Although the GPS (Global Positioning System) provides good accuracy for location, indoors it loses the direct line of sight with the satellites, degrading the accuracy. In fifth-generation mobile networks, they have features that can improve the location accuracy of the device. This work presents an approach for the pre-processing of the location in indoor environments using parameters determined from a 5G network transmission and geometric formulations of propagation of an NLoS (Non-Lineof-Sight) environment.

Keywords - Visually Impaired Persons, Localization, 5G.

\section{INTRODUÇÃO}

Segundo dados da OMS (Organização Mundial da Saúde) de 2020, aproximadamente 2,2 bilhões de pessoas em todo o mundo tem alguma deficiência visual, incluindo cegueira total ou parcial [1]. Sobre cegueira total a OMS fez um último apontamento em 2010, estimando que cerca de 39 milhões de pessoas no mundo eram completamente cegas [2]. As pessoas com deficiência visual enfrentam diversos desafios diariamente. O que para muitos é uma tarefa fácil e simples, para o deficiente visual pode se tornar um grande desafio. Um dos aspectos em que o deficiente visual tem grande dificuldade está relacionado à locomoção e localização no espaço físico, como uma ida ao banco, a uma repartição pública, visitar alguém em um edifício ou encontrar sua sala de aula em uma instituição de ensino. Há diversos desafios durante o percurso da locomoção, tanto relacionados à localização quanto a obstáculos pelo caminho. Entretanto, pontua-se a dificuldade específica quando se chega ao destino, no ambiente interno. Em uma grande faculdade, por exemplo, há diversos

Paulo Francisco da Conceição e Flávio Geraldo Coelho Rocha, Escola de Engenharia Elétrica, Mecânica e de Computação - UFG, Goiânia(GO), Brasil, e-mails: paulofrancisco@discente.ufg.br e flaviogcr@ufg.br departamentos e salas de aula, podendo haver ainda vários níveis de piso [3].

Para auxiliar na locomoção e localização é possível usar um dispositivo móvel, que esteja presente com a pessoa todo o tempo, e o smartphone cumpre bem esse papel. Diversas técnicas já foram estudadas para realizar a localização de um dispositivo no espaço físico. Uma delas (talvez a mais conhecida) é o GPS (Global Positioning System), que usa diversos satélites e fornece a localização com precisão de dezenas de metros. Entretanto, esta precisão diminui em ambientes internos [4].

Uma das alternativas ao GPS que pode ser aplicada a ambientes internos é o uso da própria rede celular, em que diversos parâmetros do sinal podem ser usados para realizar a localização, como as medidas de tempo e ângulos (ToA - Time-of-Arrival, AoD - Angle of Departure, AoA - Angle of Arrival). Sabe-se que com as medidas dos ângulos é possível calcular azimute e elevação, podendo assim localizar o dispositivo em um ambiente utilizando uma perspectiva em $3 \mathrm{D}$, determinando, por exemplo, o andar em que uma pessoa está em um edifício [5].

Sabe-se ainda que na rede celular há muitos caminhos entre o transmissor e o receptor, podendo estes caminhos terem ou não linha de visada direta entre transmissor e receptor. Em um ambiente interno a probabilidade do caminho ser sem linha de visada direta (NLoS - Non-Line-of-Sight) é muito grande e, consequentemente dificulta a localização e afeta diretamente na precisão. Entretanto, a tecnologia de redes móveis de quinta geração $(5 \mathrm{G})$ traz algumas características que melhoram muito a precisão na localização, até mesmo em ambientes internos. Dentre as principais características que impactam diretamente na localização, pode-se citar o ambiente ultradenso, em que haverá diversas antenas replicadoras que estarão mais próximas do dispositivo móvel (UDN - Ultra-Dense Network), as ondas milimétricas (mm-wave - millimeter-wave) e o número massivo de antenas no transmissor e receptor (MIMO - Multiple-Input-Multiple-Output) [6].

Assim, esse trabalho apresenta uma abordagem para o que se pode chamar de pré-processamento da localização, que é uma estimativa inicial da posição e orientação do dispositivo. Para isso serão usados minimamente parâmetros que podem ser coletados no downlink de uma possível transmissão em uma rede $5 \mathrm{G}$, usando assim a geometria de uma propagação em ambiente NLoS. A estimativa obtida com a abordagem proposta neste trabalho pode, posteriormente, ser usada como ponto de partida para uma técnica mais precisa de localização e rastreamento, como apresentado em [7], [8] e [9].

$\mathrm{O}$ restante do artigo está dividido da seguinte forma. $\mathrm{Na}$ 
Seção II são apresentados alguns trabalhos relacionados à localização. Na Seção III são descritas as formulações e modelo do sistema proposto. Na Seção IV são mostrados os resultados das simulações e na Seção V são descritas as conclusões obtidas.

\section{TRABALhos RELACIONADOS}

$\mathrm{Na}$ tentativa de localizar o dispositivo móvel, algumas pesquisas já foram realizadas envolvendo diversas técnicas. Especificamente com o intuito de auxiliar o deficiente visual, em [3] é feita uma revisão da literatura apontando algumas técnicas que podem ser usadas para localização tanto em ambiente outdoor como indoor. Dentre as técnicas recomendadas para ambientes internos estão as que envolvem ZigBee [10], Bluetooth [11] e Radio Frequency Identification (RFID) [12]. Após descrever o funcionamento de algumas técnicas, [3] propõe um método híbrido de localização envolvendo ZigBee, RFID e GPS.

Usar diversas tecnologias para realizar a localização, embora possa produzir bons resultados, pode se tornar algo complexo, podendo envolver infraestrutura por parte da instituição que desejar oferecer localização em suas instalações, e cada instituição poderia propor uma infraestrutura diferente, podendo gerar confusão para o usuário final que teria que saber o que usar dependendo de qual instituição seja seu destino. Por isso usar técnicas que usem apenas a rede celular simplifica todo o procedimento de localização.

Algumas técnicas tentam alcançar precisão usando apenas a rede celular. Em [13] e [14], os autores tentam de forma simples usar a geometria do canal para fazer uma estimativa inicial da localização do dispositivo móvel, enquanto que em [15] e [16], os autores usam filtros de Kalman e a exploração da formação de feixes, para precisar a localização do dispositivo móvel na ordem dos centímetros e fornecer também a orientação.

De acordo com [17] e [18], há ainda muito a ser explorado no que se refere à localização do dispositivo móvel usando apenas a rede celular: é necessário melhorar a precisão na localização bem como também aperfeiçoar ou desenvolver novas técnicas de localização e rastreamento, principalmente em ambiente indoor.

\section{Sistema Proposto}

No sistema de posicionamento $5 \mathrm{G}$, conforme definido em [19] e apresentado de forma resumida em [13], do sinal recebido é possível extrair informações de ângulo e tempo. De forma geral tem-se o seguinte modelo de sinal recebido:

$$
y(t)=\sum_{l=0}^{L} h_{l} a_{R}\left(\theta_{l}\right) a_{T}^{H}\left(\phi_{l}\right) S\left(t-\tau_{l}\right)+n(t),
$$

em que $y(t)$ é o sinal recebido, $S(t)$ é o sinal transmitido, $h_{l}$ é um ganho de percurso complexo, $a_{R}$ é um vetor com o par de valores do ângulo de chegada, $\theta_{l}$ é o azimute e elevação do ângulo de chegada (AoA), $a_{T}$ é um vetor com o par de valores do ângulo de partida, $\phi_{l}$ é o azimute e elevação do ângulo de partida (AoD), $\tau_{l}$ é o tempo de chegada (ToA), e $n(t)$ é um possível ruído. A quantidade de caminhos é indicada em $L$, sendo que o caminho $l=0$, indica o caminho em que há linha de visada direta (LoS - Line-of-Sight) e os demais são caminhos NLoS.

Normalmente na localização em ambientes internos haverá presença apenas de caminhos NLoS, em que há maior complexidade na precisão da localização. Entretanto é esta a situação de maior interesse para localizar e direcionar um indivíduo em ambientes indoor. Portanto, optou-se por trabalhar apenas com o cenário NLoS. Para mitigar a complexidade e aumentar a precisão na localização, espera-se que nas redes $5 \mathrm{G}$ haja um maior número de espalhadores. Com isso haverá sempre um ou mais espalhadores próximos ao receptor, fazendo com que o caminhos NLoS não tenham grandes perturbações do ambiente e tenha nível de ruído baixo [17].

Em técnicas mais precisas de localização e rastreamento, como as que usam diferença de tempo de chegada (TDoA - Time Difference of Arrival), é importante ter conhecimento prévio da localização da Estação Base (EB) e da localização dos espalhadores, que também podem ser considerados pontos de reflexão (PR). Além disso algumas técnicas exigem uma estimativa inicial da Estação Móvel (EM) para que haja convergência, como as técnicas que usam Nonlinear Least Squares [14]. Também pode ocorrer de o transmissor e o receptor não estarem sincronizados, por isso um atraso $B$ (Bias) deve ser considerado [13].

A abordagem apresentada a seguir tem por objetivo usar as informações de ângulo e tempo do sinal recebido, para então estimar a localização e orientação da EM e dos pontos de reflexão (PR). Tanto a posição da $\operatorname{EM}\left(X_{M}\right)$, da $\operatorname{EB}\left(X_{B}\right)$ e $\operatorname{dos} \operatorname{PR}\left(X_{R, l}\right)$ são $\in \mathbb{R}^{3}$. O atraso $(B)$ e a orientação para o eixo vertical $(\alpha)$ também precisam ser determinados, pois são de vital importância para a precisão da localização dentro da abordagem proposta. A figura 1 apresenta o cenário para localização proposta neste trabalho.

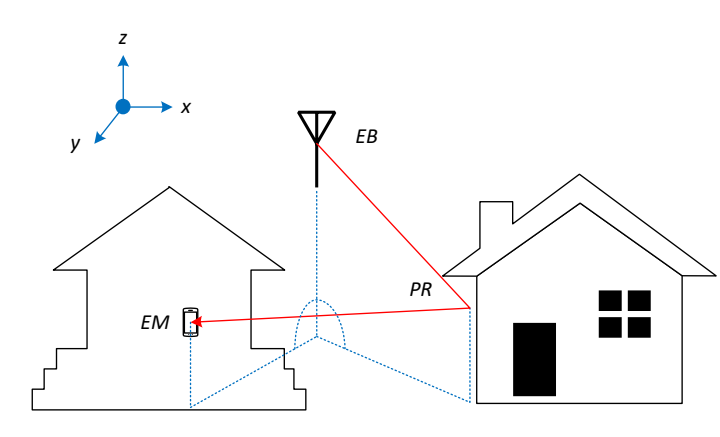

Fig. 1. Ilustração do Cenário de Localização

A seta exibida na figura 1 mostra um dos diversos caminhos NLoS possíveis. Portanto, será determinado $X_{M}, \alpha, B$ e $\left\{X_{R, l}\right\}_{l=1}^{L}$. Conforme apresentado em [13], [14] e [19], assume-se que há uma rotina de estimação dos parâmetros, que podem ser modelados a partir das seguintes formulações geométricas:

- ToA: $\tau_{l}=\left\|X_{R, l}-X_{B}\right\|+\left\|X_{R, l}-X_{M}\right\|+B$; 
- AoA - azimute: $\theta_{l}^{(a z)}=\pi+\arctan 2\left(y_{R, l}-y, x_{R, l}-\right.$ $x)-\alpha$, em que $X_{R, l}=\left[x_{R, l}, y_{R, l}, z_{R, l}\right]^{T}$ e $X_{M}=$ $\left[x_{M}, y_{M}, z_{M}\right)^{T}$;

- AoA - elevação: $\theta_{l}^{(e l)}=\arcsin \left(\left(z_{R, l}-z\right) /\left\|X_{R, l}-X_{M}\right\|\right)$;

- AoD - azimute: $\phi_{l}^{(a z)}=\arctan 2\left(y_{R, l}, x_{R, l}\right)$;

- AoD - elevação: $\phi_{l}^{(e l)}=\arcsin \left(\left(z_{R, l}-z_{B}\right) / \| X_{R, l}-\right.$ $\left.X_{B} \|\right)$, em que $X_{B}=\left[x_{B}, y_{B}, z_{B}\right]^{T}$.

As formulações relacionadas aos ângulos estão em conformidade com o sistema horizontal de coordenadas [20], conforme ilustrado na figura 2, que mostra visualmente os ângulos de um objeto em relação a um observador. Para este trabalho é considerado que o observador está localizado na origem enquanto o objeto pode ser a EB, EM ou um PR. Seguem explicações das terminologias e operações geométricas usadas:

- Azimute: É medida de abertura angular em relação ao eixo x [20];

- Elevação: É o ângulo entre o objeto em relação ao horizonte [20];

- Arctan2: Conhecida também como arco-tangente de 2 argumentos (inversa da tangente), é a operação usada para determinar o ângulo no plano euclidiano entre um ponto $(\mathrm{x}, \mathrm{y})$ e o eixo $\mathrm{X}$. Com esta operação é possível determinar o azimute [21];

- Arcsin: É a inversa da função seno, usada para calcular a elevação [21].

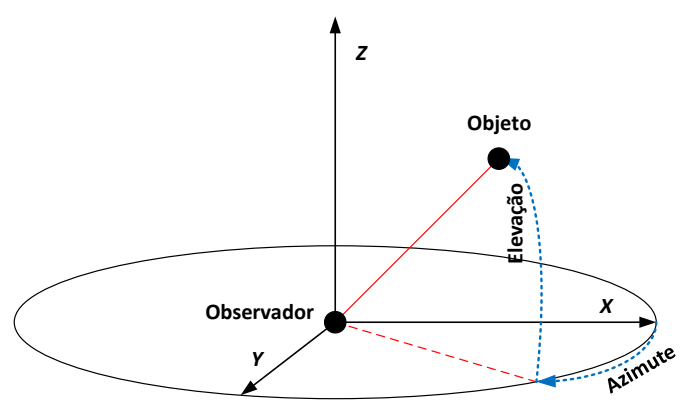

Fig. 2. Ilustração do Plano Horizontal para Determinar os Ângulos Fonte: Adaptado de [20]

Com os parâmetros obtidos é possível então determinar segmentos de linhas em que estão presentes os pontos de reflexão $\left(X_{R}\right)$ e a estação móvel $\left(X_{M}\right)$. Neste momento é importante o conhecimento, com máxima precisão possível, da orientação $(\alpha)$ e do atraso $(B)$. Primeiro determina-se $\rho=\tau_{l}-B$ e então calcula-se $s_{l}$ por meio da seguinte formulação:

$$
s_{l}=X_{B}+\rho\left[\begin{array}{l}
\cos \left(\phi_{l}^{(e l)} \cos \left(\phi_{l}^{(a z)}\right)\right. \\
\cos \left(\phi_{l}^{(e l)} \sin \left(\phi_{l}^{(a z)}\right)\right. \\
\sin \left(\phi_{l}^{(e l)}\right)
\end{array}\right] .
$$

O ponto de reflexão $\left(X_{R, l}\right)$ estará em algum ponto do segmento de linha entre $X_{B}$ e $s_{l}$.

Agora calcula-se $r_{l}$ por meio da seguinte formulação:

$$
r_{l}=X_{B}+\rho\left[\begin{array}{l}
\cos \left(\theta_{l}^{(e l)} \cos \left(\theta_{l}^{(a z)}+\alpha-\pi\right)\right. \\
\cos \left(\theta_{l}^{(e l)} \sin \left(\theta_{l}^{(a z)}+\alpha-\pi\right)\right. \\
\sin \left(-\theta^{(e l)}\right)
\end{array}\right] .
$$

A estação móvel $\left(X_{m}\right)$ estará em algum ponto do segmento de linha entre $s_{l}$ e $r_{l}$.

A figura 3 ilustra este cenário geométrico, em que $x, y$ e $z$ são as coordenadas (em metros) dos pontos, considerando 3 refletores. No cenário apresentado na figura 3 não há presença de ruídos e os parâmetros são exatos, incluindo a orientação $\alpha$ e o atraso $(B)$. Seguindo essa abordagem é possível determinar com exatidão a localização da EM, pois todos os segmentos de linha entre $s_{l}$ e $r_{l}$ cruzam no mesmo ponto, que é a localização da EM.

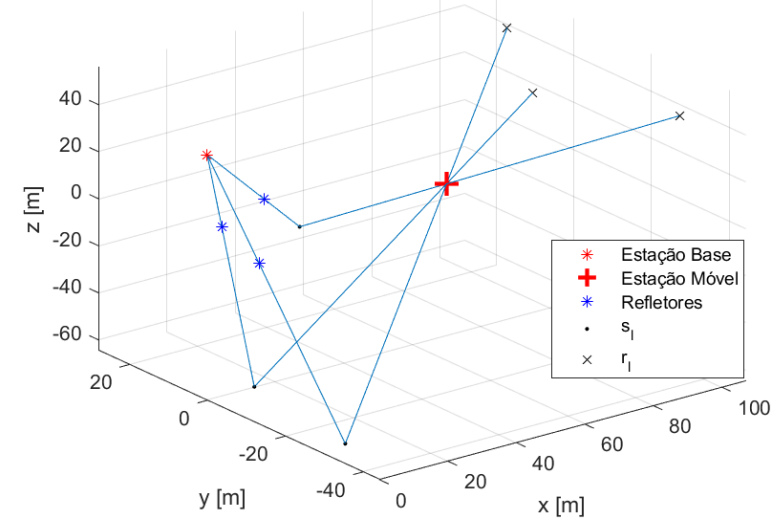

Fig. 3. Visualização das linhas, para valores corretos $(\alpha, B)$ e sem a presença de perturbações.

A figura 4 mostra o resultado quando os valores de $\alpha$ e $B$ estão incorretos, ficando então impossível determinar a localização da EM.

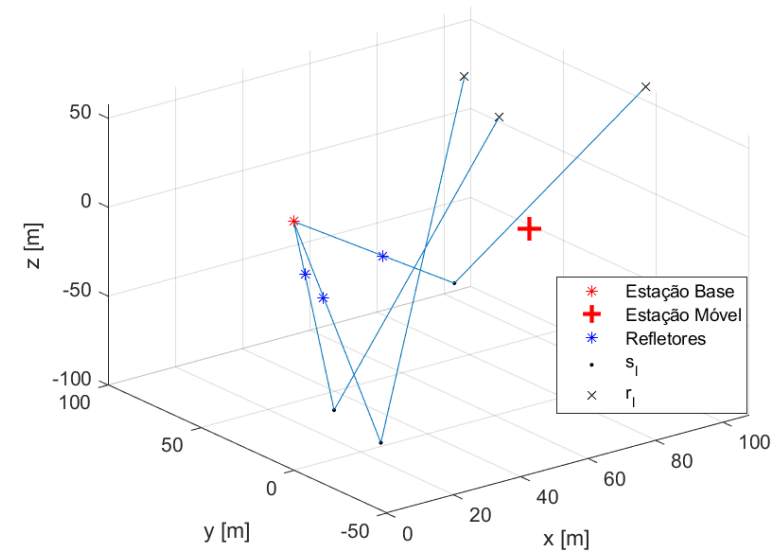

Fig. 4. Visualização das linhas, para valores incorretos $(\alpha, B)$.

Além de possíveis valores incorretos de $\alpha$ e $B$, sabe-se ainda que há presença de ruído no ambiente. Entretanto, por amostragem, é possível determinar a menor distância entre diversos segmentos de linhas $\left[s_{l}, r_{l}\right]$ para diversos valores de $\alpha$ e B. Seguindo a descrição encontrada em [13], executa-se as seguintes ações: 
1) Atribuir um valor inicial aleatório para $\alpha$ e $B$;

2) Gerar os segmentos de linhas entre os pontos $s_{l}$ e $r_{l}$ para cada amostra;

3) Determinar a distância $\left(d_{l}\right)$ entre os segmentos $\left[s_{l}, r_{l}\right]$;

4) Calcular o erro:

$$
\varepsilon(\alpha, B)=\frac{2}{N s(L(L-1))} \sum_{n=1}^{N_{s}} \sum_{l=1}^{L} \sum_{k=l+1}^{L} d_{l, k}^{(n)},
$$

em que $N_{s}$ é a quantidade de amostras.

Para determinar os melhores valores de $\alpha$ e $B$ o erro pode ser minimizado:

$$
(\alpha, B)=\arg \min _{(\alpha, B)} \varepsilon(\alpha, B) .
$$

Para minimizar $\varepsilon(\alpha, B)$, foi usado um algoritmo genérico de gradiente, conforme descrito em [22]. Assim, ao sugerir um valor inicial para $\alpha$ e $B$, seus valores ideais podem ser determinados ao encontrar o menor resultado, conforme (4).

Apos determinar os melhores valores de $\alpha$ e $B$ é possível determinar uma possível localização da EM analisando em que as linhas $\left[s_{l}, r_{l}\right]$ se cruzam, calculando a média do conjunto de amostragem da seguinte forma:

$$
\mu_{E M}=\frac{2}{N s(L(L-1))} \sum_{n=1}^{N_{s}} \sum_{l=1}^{L} \sum_{k=l+1}^{L} X_{l, k}^{(n)} .
$$

Obtida a possível localização da EM e dos valores $(\alpha, B)$, a abordagem apresentada neste trabalho difere do que é apresentado em [13] e faz uso de algumas formulações apresentadas em [14] para determinar a localização dos espalhadores, tendo em vista que as posições da EM e da EB já são conhecidas.

Primeiramente é determinada a distância entre a estação base e cada ponto de reflexão:

$$
d_{B, l}=\frac{x_{m}-x_{B}+(\tau-B) \sin \left(\theta_{l}^{(e l)}\right) \cos \left(\theta_{l}^{a z}\right)}{\sin \left(\phi_{l}^{(e l)}\right) \cos \left(\phi_{l}^{(a z)}\right)+\sin \left(\theta_{l}^{(e l)}\right) \cos \left(\theta_{l}^{(a z)}\right)} .
$$

Por último é determinada a localização de cada ponto de reflexão:

$$
\begin{aligned}
& x_{R, l}=x_{B}+d_{B, l} \sin \left(\phi_{l}^{(e l)}\right) \cos \left(\phi_{l}^{(a z)}\right), \\
& y_{R, l}=y_{B}+d_{B, l} \sin \left(\phi_{l}^{(e l)}\right) \sin \left(\phi_{l}^{(a z)}\right) \mathrm{e} \\
& z_{R, l}=z_{B}+d_{B, l} \cos \left(\phi_{l}^{(e l)}\right) .
\end{aligned}
$$

\section{Resultados e Simulações}

Para as simulações foi definido um desvio padrão de 0,01 (rad) para os ângulos e $10 \mathrm{~cm}$ para o ToA. Estes valores foram escolhidos por simular um ambiente com baixo nível ruído, conforme sugerido por [13]. O valor real de $\alpha$ foi de $\pi / 3$ e $B=10$, escolhidos arbitrariamente. A estação base está localizada na posição $(0,0,50)$ e a estação móvel na posição $(10,20,5)$, também escolhidos arbitrariamente. Foram também considerados 4 pontos de reflexão em locais aleatórios, distribuídos uniformemente entre -50 e 50 para cada eixo $(x, y$ e $z)$. Após alguns testes sobre a quantidade de amostras suficientes para produzir resultados satisfatórios, optou-se por usar 20 amostras em cada simulação. Com estas configurações e usando valores arbitrários iniciais para $\alpha$ e $B$, o gráfico de minimização do erro foi gerado e pode ser visto na figura 5 .

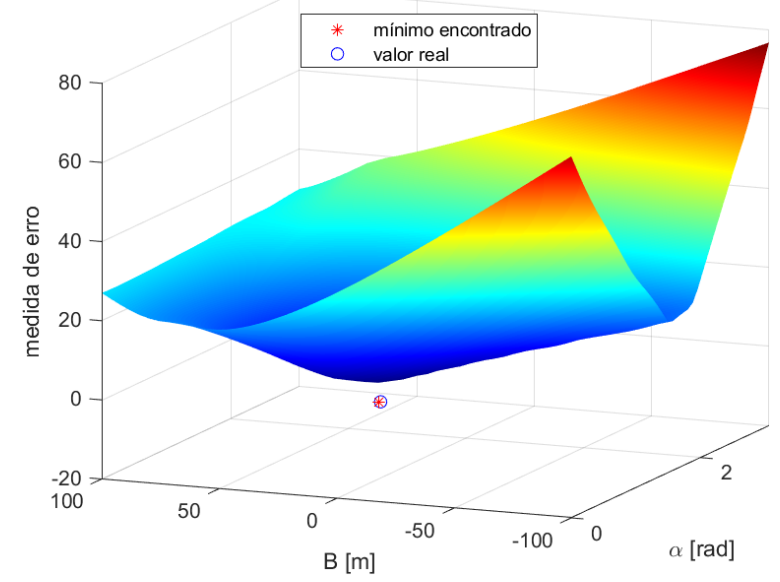

Fig. 5. Gráfico da minimização do erro para $(\alpha, B)$.

Foram feitas 1000 simulações, entretanto, tendo em vista que a localização dos espalhadores é gerada aleatoriamente em cada simulação, foi escolhida uma das simulações, de forma aleatória, para produzir a figura 6 que mostra o cenário hipotético final. Para esta simulação é possível perceber que a técnica estima com sucesso a localização da EM e de todos pontos de reflexão. Na figura 6 , há um pequeno segmento de linha entre o • (localização real) e o * (localização estimada).

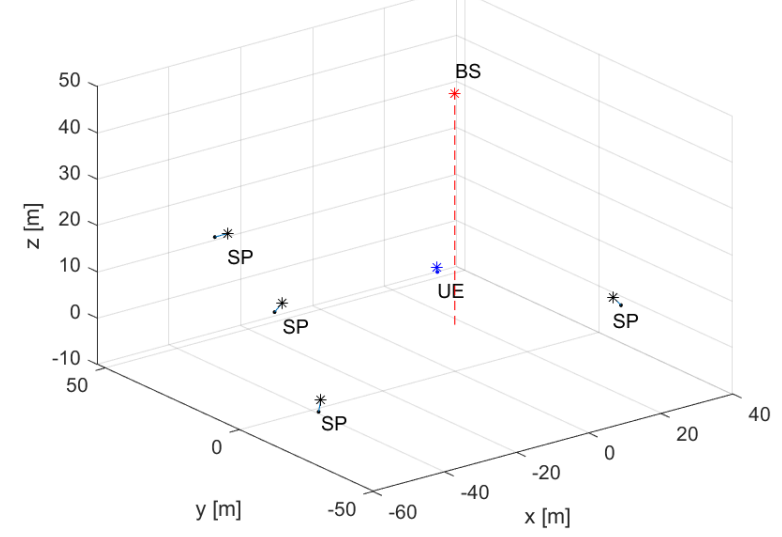

Fig. 6. Resultado de uma das simulações.

Para melhor avaliar a precisão da técnica, para as 1000 simulações, foi calculada a média do EQM (Erro Quadrático Médio) referente à localização da EM e dos pontos de reflexão, de tal forma que $E Q M_{X_{M}}=\left\|X_{M}-\widehat{X}_{M}\right\|$ e $E Q M_{X_{R}}=$ $\left\|X_{R}-\widehat{X}_{R}\right\|$, em que $\widehat{X}_{M}$ e $\widehat{X}_{R}$ se referem aos valores estimados para EM e PR, respectivamente. A probabilidade de acerto na localização da estação móvel é mostrada na figura 7 e a probabilidade de acerto para a localização dos espalhadores é mostrada na figura 8. As figuras 7 e 8 mostram no eixo y o valor do EQM para cada simulação ordenados de forma crescente, mostrando assim no eixo x a probabilidade 
de acerto. A média do EQM para a estação móvel foi de 5, 08 (m) e dos pontos de reflexão foi de 2,25 (m).

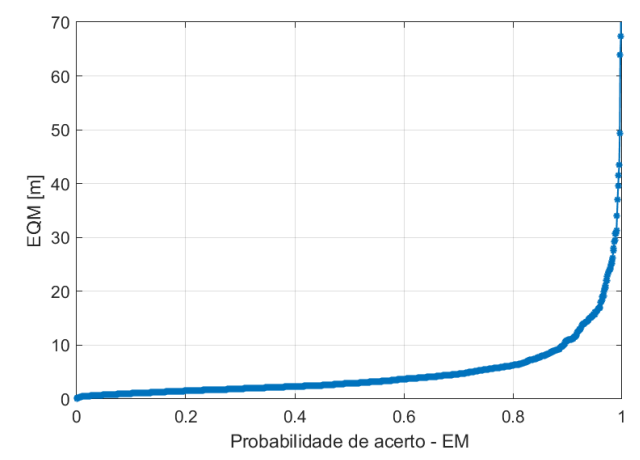

Fig. 7. EQM para localização da EM.

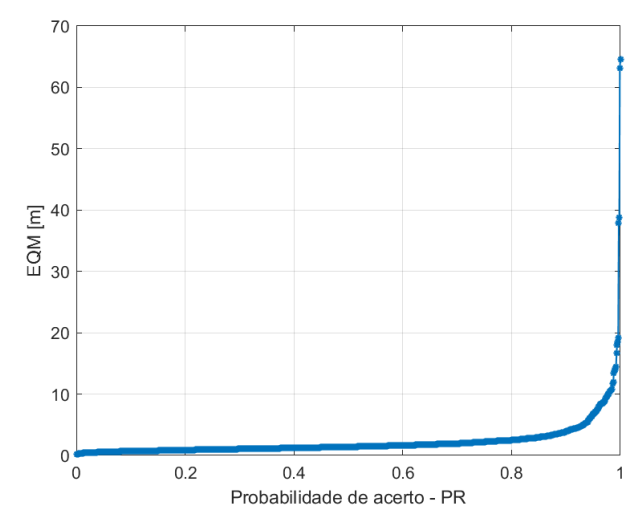

Fig. 8. EQM para localização dos PR's.

\section{CONCLUSÃO}

Este trabalho apresentou uma abordagem para determinar a posição dos espalhadores e da estação móvel em um cenário NLoS, usando parâmetros da rede móvel. O cenário NLoS foi escolhido pois pretende-se aplicar a localização do dispositivo móvel para guiar pessoas com deficiência visual em ambientes internos, como uma universidade.

No ambiente interno há vários pontos de reflexão e as posições destes pontos são desconhecidas. Assim foi usada a geometria do canal para traçar segmentos de retas e estimar a posição da estação móvel e dos pontos de reflexão. E Erro Quadrático Médio foi usado como medida de desempenho e foram encontrados os valores $2,25(\mathrm{~m})$ para a localização dos espalhadores e 5, 08 (m) para localização da EM. Estes valores podem ser considerados altos para um ambiente interno, em que é necessário uma precisão na ordem dos centímetros.

Portanto, a técnica pode ser usada como pré-processamento de uma abordagem mais complexa de localização, que normalmente exige um valor inicial para a localização que seja próximo do valor real, de forma que garanta convergência. Assim, pretende-se dar continuidade a este trabalho buscando realizar um ajuste na precisão da localização obtida, visando a ordem dos centímetros, para que então seja realmente factível usar apenas a rede celular no auxílio da localização e contribuir para uma melhor qualidade de vida da pessoa com deficiência visual.

\section{REFERÊNCIAS}

[1] O. M. da Saúde., "Relatório Mundial sobre a Visão - 2021". https://www.who.int/publications-detail/world-report-on-vision, acesso em 26/04/2021, 2021.

[2] W. H. Organization., "Global Data on Visual Impairments - 2010". https://www.who.int/blindness/GLOBALDATAFINALforweb.pdf?ua=1, acesso em 15/05/2021, 2012.

[3] R. D. Al-kafaji, S. K. Gharghan, e S. Q. Mahdi, "Localization techniques for blind people in outdoor/indoor environments: Review," IOP Conference Series: Materials Science and Engineering, vol. 745, p. 012103, Março 2020. [Online]. Available: https://doi.org/10.1088/1757$899 \mathrm{x} / 745 / 1 / 012103$

[4] K. Liu, H. B. Lim, E. Frazzoli, H. Ji, e V. C. S. Lee, "Improving positioning accuracy using gps pseudorange measurements for cooperative vehicular localization," IEEE Transactions on Vehicular Technology, vol. 63, no. 6, pp. 2544-2556, 2014.

[5] Y. Zhao, Z. Li, B. Hao, P. Wan, e L. Wang, "How to select the best sensors for tdoa and tdoa/aoa localization?" China Communications, vol. 16, no. 2, pp. 134-145, 2019.

[6] Y. Wang e K. C. Ho, "Tdoa positioning irrespective of source range," IEEE Transactions on Signal Processing, vol. 65, no. 6, pp. 1447-1460, 2017.

[7] A. Shahmansoori, G. E. Garcia, G. Destino, G. Seco-Granados, e H. Wymeersch, "Position and orientation estimation through millimeter-wave mimo in 5g systems," IEEE Transactions on Wireless Communications, vol. 17, no. 3, pp. 1822-1835, Março 2018.

[8] J. García-Fernández, A. Jurado-Navas, M. Fernández-Navarro, e C. Úbeda, "A comparative study between iterative algorithms for tdoa based geolocation techniques in real umts networks," Mobile Networks and Applications, vol. 25, no. 4, pp. 1572-8153, 2020.

[9] Y. Lu, M. Koivisto, J. Talvitie, M. Valkama, e E. S. Lohan, "Ekf-based and geometry-based positioning under location uncertainty of access nodes in indoor environment," em 2019 International Conference on Indoor Positioning and Indoor Navigation (IPIN), 2019, pp. 1-7.

[10] H. Jawad, R. Nordin, S. Gharghan, A. Jawad, e M. Ismail, "Energyefficient wireless sensor networks for precision agriculture: A review," Sensors, vol. 17, p. 1781, Agosto 2017.

[11] L. Wan, G. Han, L. Shu, e N. Feng, "The critical patients localization algorithm using sparse representation for mixed signals in emergency healthcare system," IEEE Systems Journal, vol. 12, no. 1, pp. 52-63, 2018.

[12] C. Tsirmpas, A. Rompas, O. Fokou, e D. Koutsouris, "An indoor navigation system for visually impaired and elderly people based on radio frequency identification (rfid)," Information Sciences, vol. 320, pp. 288-305, 2015. [Online]. Available: https://www.sciencedirect.com/science/article/pii/S0020025514008007

[13] H. Wymeersch, "A simple method for $5 \mathrm{~g}$ positioning and synchronization without line-of-sight," arXiv preprint arXiv:1812.05417, 2018.

[14] B. Y. Shikur e T. Weber, "Tdoa/aod/aoa localization in nlos environments," em 2014 IEEE International Conference on Acoustics, Speech and Signal Processing (ICASSP), 2014, pp. 6518-6522.

[15] E. Rastorgueva-Foi, M. Costa, M. Koivisto, J. Talvitie, K. Leppaneny, e M. Valkama, "Beam-based device positioning in mmwave $5 \mathrm{~g}$ systems under orientation uncertainties," em 2018 52nd Asilomar Conference on Signals, Systems, and Computers, 2018, pp. 3-7.

[16] E. Rastorgueva-Foi, M. Costa, M. Koivisto, K. Leppanen, e M. Valkama, "User positioning in mmw $5 \mathrm{~g}$ networks using beam-rsrp measurements and kalman filtering," em 2018 21st International Conference on Information Fusion (FUSION), 2018, pp. 1-7.

[17] Q. Liu, R. Liu, Z. Wang, e Y. Zhang, "Simulation and analysis of device positioning in 5g ultra-dense network," em 2019 15th International Wireless Communications Mobile Computing Conference (IWCMC), 2019, pp. 1529-1533.

[18] F. Wen, H. Wymeersch, B. Peng, W. P. Tay, H. C. So, e D. Yang, "A survey on $5 \mathrm{~g}$ massive mimo localization," Digital Signal Processing, vol. 94, pp. 21-28, 2019, special Issue on Source Localization in Massive MIMO. [Online]. Available: https://www.sciencedirect.com/science/article/pii/S1051200419300569

[19] 3rd Generation Partnership Project, Technical Specification Group Radio Access Network - Release 16. http://www.3gpp.org, acesso em 20/04/2021, 2019.

[20] J. Sickle, "Basic GIS Coordinates - 3rd Edition". CRC Press, 2017.

[21] R. Larson, Algebra \& Trigonometry. Cengage Learning, 2016.

[22] R. L. Burden e J. D. Faires, Numerical Analysis - Ninth Edition. Brooks/Cole, Cengage Learning, 2011. 